\title{
CONSIDERATIONS CLINIQUES SUR L'EMPLOI DE DEUX NOUVEAUX CURARISANTS DE SYNTHESE. LE BREVATONAL ET LE MEDIATONAL
}

\author{
Eugene Allakd, M.D. et J. G. Patoine, M.D. *
}

LE Brevatonal et le Médratonal sont deux curarisants de synthèse mis sur le marché européen en 1952 mais encore très peu utlisés en Amérique du Nord, à notre connaissance du moins. Cependant, ils ont été le-sujet de nombreuses recherches tant expérimentales que cliniques en Belgique, en France et en Itahe.

Le Brévatonal est considéré comme un leptocurare bien qu'il ait une action plus tardive et plus prolongée que celle de la succinylcholine. Il a un temps de latence de 30 secondes à 3 minutes, une durée d'action de 20 à 30 minutes. Les phénomènes de curarisation passent par les étapes habituelles, il n'aurat nı effet central, nı effet histammicue, ni effet muscarınıque si ce n'est de la sialorrhée et de la bronchorrhée occasionnelles qui apparaissent surtout à l'occasion des doses insuffisantes de curarısant Il est dénué de toxicité et ne précipite pas lorsqu'on le mélange au penthiobarbital bien que l'on recommande de l'adminstrer isolément et quelques minutes avant le penthiobarbital afin de faire coincider l'action maximum de chacun si l'on doit pratiquer l'intubation Il n'est pas cumulatof et on peut répéter l'admmistration de doses efficaces au besoin

Le Médıatonal s'apparente chımıquement au Brévatonal mais il en diffère considérablement dans ses effets. Il semble tenir à la fois du curarimimétıque et de l'acétylcholimimétıque puisqu'il associe les caractéristiques de l'un et de l'autre. Aınsi, il potentralıse les leptocurares, mais il possède certains effets neurovégétatıfs à l'mstar des pachycurares, et pourtant, selon la plupart des auteurs, la prostıgmine le potentalıse au heu d'en réduire l'action. C'est pourquoí, à la suite de Brodowski et Huguenard, plusieurs auteurs le consıdèrent comme un curarisant intermédiaire unique Nous pouvons résumer les caractéristiques cliniques du Médıatonal en affirmant qu'il est doué d'un pouvoir potentialisateur extraordınarre. ll potentialıse le thiobarbital fortement, les leptocurares à 300 pour cent et les pachycurares à 1000 pour cent. Il n'est paș toxıque aux doses recommandées, soit à 30 à $40 \mathrm{mg}$ intravemeux pour l'adulte sain mais $1 \mathrm{l}$ est fortement cumulatif sı adrninistié à moins de 30 minutes d'intervalle. Ce pouvoir potentralısateur est mis à profit dans l'association Brévatonal-Médiatonal. En effet, on peut, selon cette technique, réduire très considérablement et la quantité et la fréquence des doses de curarısants et obtenir quand même un relâchement musculaire prolongé tout en déprimant très peu la respiration.

Depuis quelque six moıs, grâce à la générosité de l'Union Chimique Belge, nous avons eu l'opportunité d'olsserver les effets du Brévatonal et du Médiatonal dans un petite proportıon de nos anesthésies pratıquées à l'Hopital de l'EnfantJésus Aux fins de la présente étude, nous avons revu sans sélection ni exclusion aucune nos 106 premiers cas.

La nomenclature de ces 106 cas (tableau I) représente assez fidèlement la

"Service d'Anesthésie de l'Hopital de l'Enfant-Jésus, Québec Travarl présenté le 5 ma1 à l'Hopital Laval, à une réunion de la Société Canadienne d'Anesthésıe, Section de Québec. 
proportion habituelle des interventions couramment pratiquées dans nos milieux, exception faite de certaines qui requièrent une technique particulière comme les césariennes, les interventions de neurochirurgie et beaucoup d'autres faites sous anesthésie rachidienne.

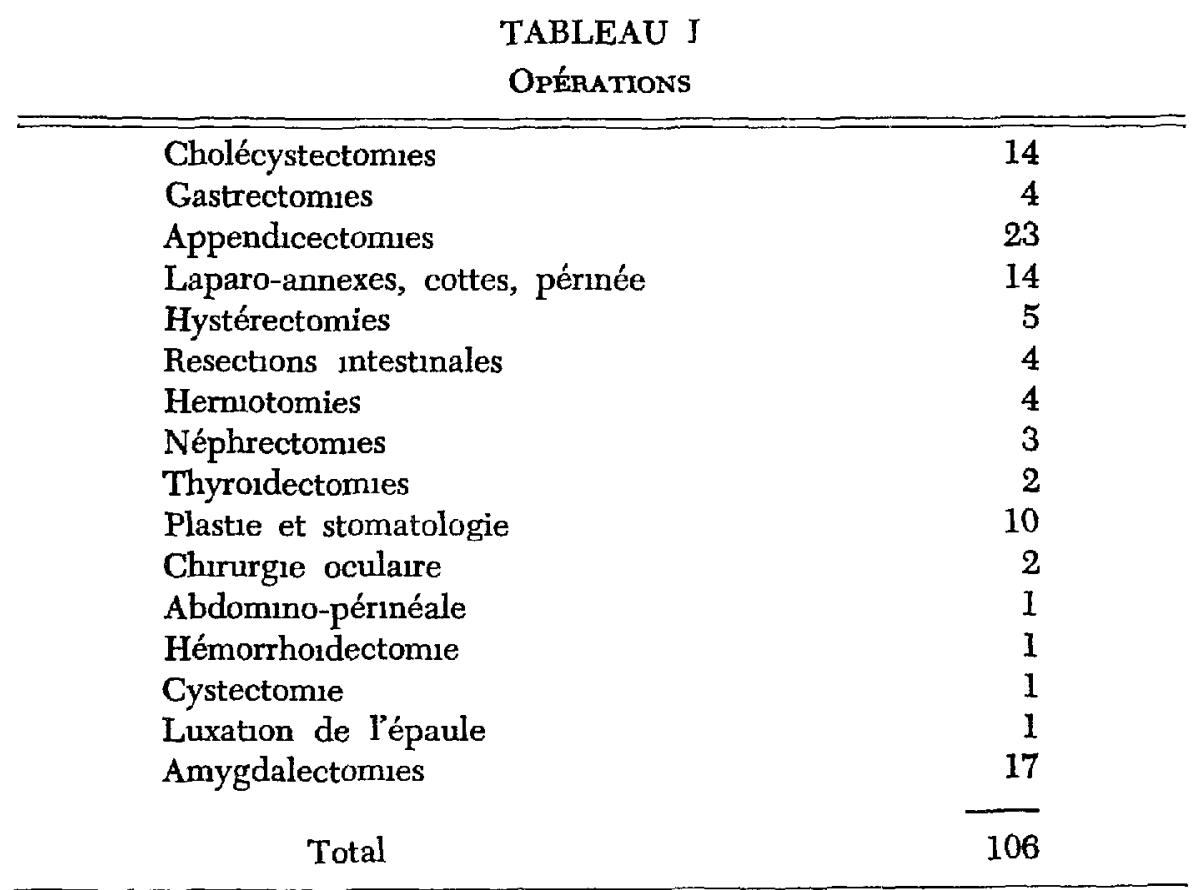

Tableau II présente quelques détails sur les techniques suivies au cours de ces interventions. Dans tous les cas, nous avons pratiqué linduction au Pentothal à la dose moyenne de $480 \mathrm{mg}$, après préparation préanesthésique au Nembutal ou Séconal, Démérol et Atropine. Nous avons utilisé le Brévatonal seul dans 65 cas, à la dose moyenne de $4.7 \mathrm{mg}$., le Médiatonal seul dans 13 cas à la dose moyenne de $19 \mathrm{mg}$. et l'association des deux dans 28 cas à la dose moyenne respective de $4 \mathrm{mg}$. et de $9.2 \mathrm{mg}$.

TABLEAU II

Agents anesthísiques et curarisants

\begin{tabular}{|c|c|c|c|c|}
\hline & $\begin{array}{c}\text { Nombre de } \\
\text { cas }\end{array}$ & $\begin{array}{c}\text { Dose max } \\
\text { mg }\end{array}$ & $\begin{array}{c}\text { Dose min } \\
\text { mg; }\end{array}$ & $\begin{array}{c}\text { Dose moyenne } \\
\text { mg }\end{array}$ \\
\hline Pentothal (Induction) & 106 & 1000 & 100 & 480 \\
\hline Brévatonal & 65 & 10 & 1.5 & 47 \\
\hline Médiatonal & 13 & 35 & 5 & 19 \\
\hline $\begin{array}{l}\text { Association } \\
\text { Brévatonal } \\
\text { Médiatonal }\end{array}$ & 28 & $\begin{array}{l}10 \\
20\end{array}$ & $\begin{array}{l}2 \\
3\end{array}$ & $\begin{array}{l}4 \\
92\end{array}$ \\
\hline $\mathrm{N}^{2} \mathrm{O}+\mathrm{O}^{2}$ & 84 & & & \\
\hline $\mathrm{N}^{20}+\mathrm{O}^{2}+$ éther & 3 & & & \\
\hline $\mathrm{C}^{3} \mathrm{H}^{6}+\mathrm{O}^{2}$ & 20 & & & \\
\hline $\mathrm{O}^{2}$ seul & 2 & & & \\
\hline
\end{tabular}


L'anesthésique complémentaire a été le protoxyde d'azote et oxygène dans 87 cas et le cyclopropane-oxygène dans 20 cas et de l'oxygène seul dans deux cas.

Sans tenir compte de la durée des interventions ou du dégré de relâchement musculaire nécessarre, on constate dès maintenant que dans 81 pour cent des cas le protoxyde d'azote et oxygène associés à une très petite dose de Pentothal et aux myorésolutifs Brévatonal-Médıatonal, ont suffi pour maintenur une anesthésie adéquate.

Les appendicectomies, les amygdalectomies et les cholécystectomies (tableau III) sont les opérations ou nous avons le 'plus souvent employé le Brévatonal ou le Médiatonal et ceci pour deux rassons. (1) parce que ce sont là des interventions fréquentes; (2) parce que insensiblement, sans nous en rendre bien compte, nous avons eu tendance à utılsser le Brévatonal et Médiatonal, là où ils rendaient le plus service.

TABLEAU III

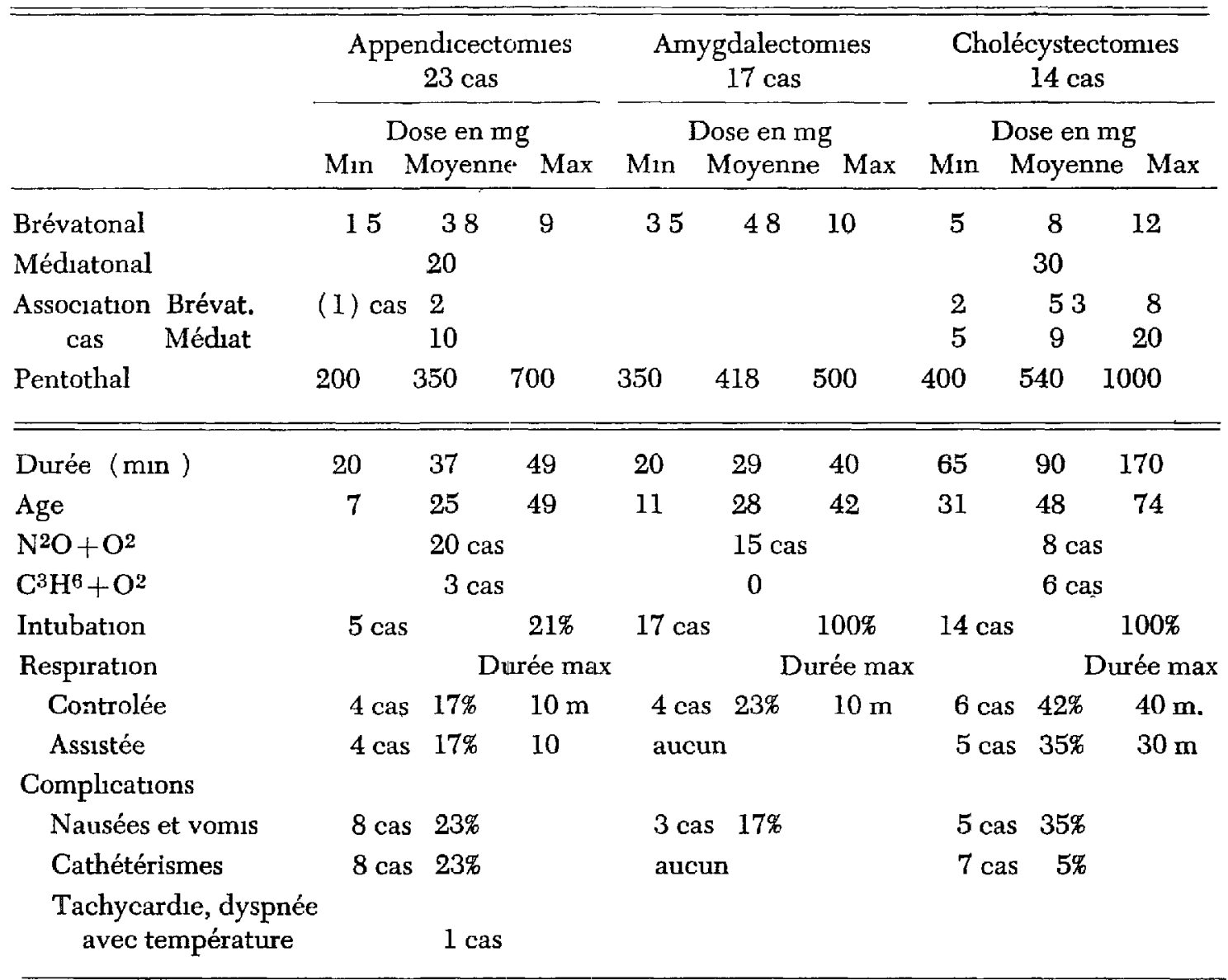

Appendicectomies Nous avons eu 23 appendicectomies dont l'âge varie de 7 à 49 ans (moyenne 25 , ans) et la durée de 20 à 75 minutes (moyenne 37 ). Ils ont reçu de 200 à $700 \mathrm{mg}$ de Pentothal (moyenne $350 \mathrm{mg}$ ), $\mathrm{N}^{2} \mathrm{O}$ et $\mathrm{O}^{2}$ dans 20 cas, $\mathrm{C}^{3} \mathrm{H}^{6}$ et $\mathrm{O}^{2}$ dans 3 cas. Cinq ont été intubés soit 21 pour cent. Dans 20 cas sur 23 nous avons donné le Brévatonal seul à la dose de 1.5 à $9 \mathrm{mg}$. (moyenne 
$3.8 \mathrm{mg}$ ), dans 2 cas le Médiatonal seul à 20 et $25 \mathrm{mg}$. Une seule fors nous avons associé $2 \mathrm{mg}$ de Brévatonal et $10 \mathrm{mg}$ de Médiatonal.

Nous avons dû contrôler la respiration dans 4 cas soit 17 pour cent pendant un maximum de 10 minutes et l'assister dans 4 autres cas pendant un maximum de 10 minutes également. Dans les suites opératoires nous avons noté des nausées et vomissements 8 fois, de la rétention urinaire 8 fois et une fois une épisode de tachycardie avec dyspnée et hyperthermie qui a d'allleurs régressé seule et qui est difficlement imputable à la curarisation

Nous avons été impressionné au cours de ces appendicectomies du relâchement abdominal parfait sans dépression respiratoire importante, c'est une constatation qui contraste un peu avec ce que nous avons obtenu avec les autres curares et que nous semble digne de mention. L'unique fors que nous avons associé le Brévatonal au Médiatonal, association que nous jugeons maintenast superflue pour une appendicectomie, il nous a fallu contrôler la respiration pendant 10 minutes alors que l'opération en a duré 50 Il est également bon dé noter que, malgré une prémédıcation plutot légère, une très fauble dose de Pentothal associé au protoxyde d'azote a suffi pour des interventions dont la durée moyenne a été de 37 minutes.

Amygdalectomies. Nous avons fart 17 cas d'amygdalectomies en nous servant du Brévatonal pour l'intubation à la dose moyenne de $48 \mathrm{mg}$ La durée moyenne a été de 29 minutes L'âge variait de 11 à 42 ans Tous les patients ont été intubés après avoır reçu du Pentothal à la dose moyenne de $418 \mathrm{mg}$. et l'anesthésıe a été complétée au protoxyde d'azote et oxygène Nous avons contrôlé la respiration dans 4 cas, solt 23 pour cent pour un maximum de 10 minutes, 3 patients, soit 17 pour cent ont présenté des nausées ou vomissements dans les heures suvantes Au cours des amygdalectomıes, nous avons été frappés d'une part dę la facilité de l'untubation, même après avorr administré la plupart de temps que la moitié de la dose de Brévatonal recommandée, d'autre part, du peu de dépression respuratoire. Le retour des réflexes est partıcuhèrement précoce en fin d'interventoon et le réveil est immédiat.

Cholécystectomies. Enfin, nous avons pratiqué 14 anesthésies pour cholécystectomies dont 5 au Brévatonal seul à la dose moyenne de $8 \mathrm{mg}$. (5 -12), une au Médiatonal seul à la dose de $30 \mathrm{mg}$. et 7 avec l'association des deux avec une moyenne respective de $53 \mathrm{mg}$ et $9 \mathrm{mg}$. Tous les patients ont été intubés La durée moyenne a été de 90 minutes et 6 patients, 42 pour cent ont reçu une respiration contrôlée pendant un maximum de 40 minutes et 5 cas, 35 pour cent une respiration assistée pendant une durée max de 30 minutes

Dans les suites opératorres, 5 malades ont présenté des nausées ou vomissements et 7 ont du être cathétérisés

Nous avons été vraiment étonnés de constater que l'atonie des muscles de l'étage supérieur de l'abdomen pouvart persister aussi longtemps avec une seule dose ou de petites doses répétées de Brévatonal que, nous avons employé seul dans cinq cas. Les autres patients ont reçu une association de Brévatonal et de Médiatonal et aucune dépression respiratoire ne s'est prolongée au point d'assister le respiration jusqu'à la fin de l'intervention. La quantité de Penthothal a êté relativement faible et l'anesthésie a été complètée au protoxyde d'azote dans 8 cas et au cyclopropane dans 6 cas 
TABLEAU IV

INCIDENCE RESPIRATOIRE

\begin{tabular}{|c|c|c|c|c|c|c|c|}
\hline & \multirow{2}{*}{$\begin{array}{c}\text { Nombre } \\
\text { de cas }\end{array}$} & \multicolumn{3}{|c|}{ Controlée } & \multicolumn{3}{|c|}{ Assisfée } \\
\hline & & Nombre & $\overline{y_{0}}$ & Durée & Nombre & $\mathscr{D}$ & Durée \\
\hline Brévatonal & 65 & 13 & 20 & 14 & 8 & 123 & 10 \\
\hline Médıatonal & 13 & 1 & 77 & 15 & 3 & 23 & 25 \\
\hline \multicolumn{8}{|l|}{ Association } \\
\hline $\begin{array}{l}\text { Brévatonal } \\
\text { Médiatonal }\end{array}$ & 28 & 12 & 428 & 30 & 14 & 50 & 18 \\
\hline Total & 106 & 26 & 246 & 20 & 25 & 24.5 & 18 \\
\hline
\end{tabular}

Une étude de tableau IV nous permet de dédure que l'un ou l'autre de ces curarisants, employé seul, modifie peu la respiration mais que l'association des deux, même à dose très réduite, aboutıt fréquemment à un arrêt respiratoire mais d'assez courte durée. Le chuffres de 20,77 et 42.8 pour cent pour la respiratıon controlée et de $12.3,23$ et 50 pour cent pour la respiration assistée en sont des preuves évidentes. Cette brıèveté d'atteinte des phénomènes respiratores compense largement pour l'absence d'un antagoniste pharmacologique de ces deux produits.

Nous n'avons jamass observé de trémulations musculaires ou le tremblement caractérıstique que l'on rencontre au moment de la dépolarısation avec le 'succinylcholıne. Même si on injecte le Brévatonal avant le Pentothal, il est très rare que les patıents se plaignent de sensation désagréable.

La sialorrhée pre-opératorre parfors rencontrée avec l'association des deux ne nous a pas semblé un inconvén ient sérieux mais la forte incıdence de rétention urnaire post-opératoire pourrait nous en faure restreindre l'usage chez les malades avec pathologie urinaire

Pour ce qui est du Médıatonal seul, nous consıdérons qu'il a des indications très restremtes puisqu’il faut en admmstrer des doses très élevées pour obtenur un relâchement musculaire efficace. Par contre le Brévatonal occupe une place avantageuse entre les curares à action très brève et les curares à action prolongée. L'intubation est toujours facıle, sans ıéflexes, avec une glotte béante moyennant que l'on attende 2 à 3 minutes av ant de procéder.

L'association Brévatonal-Méduatonal, dans les interventions de moyenne et de longue durée, ne présente pas d'avantages appréciables sur les autres curares mass elle permet de réduire considérablement les doses respectives des curarısants et aussi des substances anesthésiques complémentaires.

En conclusion, nous ne saurions affirmer la nécessité du Médiatonal d’après le pett nombre d'observations faites mass par contre nous reconnaissons une place de chorx au Brévatonal, en comblant une lacune de puissance et de temps dans la gamme des curarisants, pour les interventions de durée moyenne, toutes les fors qu'un effet myorésolutif s'impose solt pour intubation solt pour les opérations abdominales ou autres. 


\section{SUMmaRY}

Brevatonal and Mediatonal are two synthetic curare-like preparations used in Europe since 1952.

Brevatonal belongs to the leptocurare group, but compared to succinylcholine its action is slower and more prolonged. Its latent period is from one to three minutes, and its average duration is twenty minutes. It combines with thiobarbital without causing precipitation, and repeated doses do not have cumulative effect.

Mediatonal has a rapid and prolonged action. It brings into play, by trigger action, changes in the electrical potential of thiobarbital, the leptocurares to the extent of 300 per cent, and the pachycurares 1000 per cent. It is non-toxic, but has a cumulative action if the doses are repeated within thrrty-minute intervals.

The electrical potential mediator property is used in the Brevatonal-Mediatonal combination in order to reduce, to an appreciable extent, the dosage of the curare-like substance, as well as the anaesthetics.

We have used these preparations 106 times for various operative procedures. Special attention was given to ther use in the more common types of operations, such as cholecystectomies, appendectomies, and tonsillectomies. There are certain features which are worth noting.

A depolarization block due to Brevatonal is never accompanied by much fasciculation, even when administered prior to Pentothal induction. Respiratory depression was never very marked in our experience; and intubation was never a problem. In our opinion, this relaxant, as a curare-like agent of relatively long duration, should occupy an intermediate place between succinylcholine and decamethonium.

With Mediantonal alone, satisfactory muscular relaxation can be obtained with a fairly large dose; the association of Brevatonal and Mediatonal, however, procures long anaesthesia with relatively small doses of the curare-lıke substance when complementary anaesthetics are used.

Brevatonal fills a gap in the duration pattern, between succinylcholine and the curare-like substances of longer duration, it is admirably suited for operations that are neither too long nor too short, and especially suitable when intubation or muscular relaxation are required.

\section{BIBLIOGRAPHIE}

1 Brodoska et Huguenard Une association curarisante potentialısée Brévatonal-Mediatonal Anesthésie et analgésie 10 (juin 1953)

2. - - Note complémentaire au sujet d'une association curćnsante Anesthésie et analgésıe 10 (décembre 1953)

3 LADA T. Expérience personnelle sur l'emplol en anesthésiologie de deux nouveaux curarisants de synthèse, Brévatonal-Médiatonal Anesthésıe et analgésıe 11 (juin 1954).

4. Tisthoud Considérations sur un curarisant à action brève, le Brévatonal Anesthésie et analgésie 10 (décembre 1953).

5 Mundeler et LÉvis. Essa1s d'un nouveau curarisant de synthèse Acta anaesthésia belgica, No 1 (1952)

6 Mundeler. Etude clinique d'un nouveau curansant. Acca anaesthésı belgıca, No 2-3 (1952)

7 Dauni et Caporale. Le Dichlorure de 5-5 bis-triméthylarnmonium dipentyl éther comme curarisant en anesthésiologie Minerva anesthésiologica, No 8 (1954). 